\title{
La tercera generación de regulaciones de la educación superior a distancia en América Latina
}

\author{
A terceira geração de regulamentos de educação superior a \\ distância na América Latina
}

The third generation of distance education regulations in Latin America

\section{Claudio Rama*}

\section{Resumen}

El presente artículo constituye un análisis comparativo de las nuevas normativas de regulación de la educación superior a distancia en América Latina, desarrolladas desde el año 2010, en el cual se destacan especialmente las de Ecuador, Argentina, Perú, Brasil, Paraguay, Costa Rica y El Salvador. El trabajo propende a evaluar las tendencias generales de evolución de los marcos normativos a distancia, mostrando las dualidades de avances

CR: PhD, e-mail: claudiorama@gmail.com 
y retrocesos, e identificando la nueva inclusión de ofertas $100 \%$ virtuales y de sistemas más flexibles en su gestión. El artículo busca identificar los ejes comunes y diferenciadores de estas normativas de tercera generación y asociado a ello de la diferenciación de sus sistemas de educación superior y realiza una clasificación de las fases de las normativas de la educación a distancia en la región.

Palabras-clave: Educación a distancia. Educación virtual. Universidad. Regulación. América Latina.

\title{
Resumo
}

Este artigo é uma análise comparativa das novas regras que regulam a distância Ensino Superior na América Latina, desenvolvido desde 2010, em que se destacam especialmente os do Equador, Argentina, Peru, Brasil, Paraguai, Costa Rica e O salvador. O trabalho tende a avaliar as tendências gerais de evolução dos marcos regulatórios distância, mostrando as dualidades de avanços e retrocessos, e identificando a nova inclusão do virtual e oferece $100 \%$ mais flexível em seus sistemas de gestão. O artigo procura identificar eixos comuns e diferenciação destes regulamentos associados com terceira geração e esta diferenciação dos seus sistemas de ensino superior e executa uma regulamentos de classificação de fase de ensino à distância da região.

Palavras-chave: Educação a distância. Educação virtual. Universidades. Regulação. América Latina.

\begin{abstract}
This article is a comparative analysis of the new regulatory regulations for distance higher education in Latin America, developed since 2010, in particular those of Ecuador, Argentina, Peru, Brazil, Paraguay, Costa Rica and The Savior. The work tends to evaluate the general evolution trends of remote regulatory frameworks, showing the dualities of progress and setbacks, and identifying the new inclusion of $100 \%$ virtual offers and more flexible systems
\end{abstract}


in their management. The article seeks to identify the common and differentiating axes of these third generation regulations and associated with it of the differentiation of their higher education systems and makes a classification of the phases of the regulations of distance education in the region.

Keywords: Distance education. Virtual education. University. Regulation. Latinamerica.

\section{La lógica de la regulación y la expansión normativa de la educación a distancia}

La regulación, como obligación o prohibición de hacer o de límites en la libertad de hacer, tiene múltiples causas históricas, políticas, legales y culturales. Y también múltiples soportes conceptuales, desde la ética del accionar, el establecimiento de estándares mínimos para proteger al consumidor o limites al "problema del polizón", la protección social o de los ciudadanos o consumidores u orientar las actividades públicas o privadas hacia determinada orientación de las ofertas. Uno de sus basamentos más profundo es económico, en tanto algún oferente puede ser deshonesto en la competencia y no siempre los consumidores están completamente informados o, incluso, capacitados para acceder a manejar la información. Reducir las asimetrías informacionales es en este sentido una de las funciones más importante de la regulación en dinámicas donde hay múltiples actores de mercado.

En éste sentido, la existencia de "fallas de mercado" que requieren ser subsanadas, constituye el eje que ha llevado a economistas y gobiernos, a plantear la necesidad de la intervención pública en la educación superior con el objetivo de establecer criterios y limitaciones para permitir cumplir el derecho de acceso a la educación, tanto de primera, de segunda como de tercera generación en la definición de Bates (2000). Tales enfoques jurídicos asociados a los equilibrios entre las diversas 
generaciones de los derechos humanos, fijarían el marco, tanto de la libertad como de la regulación, a escala nacional e internacional, como centros gravitatorios de la política pública, y a partir de las cuales se establecen los estándares de su funcionamiento.

En general, en educación superior, la regulación se ha posicionado fundamentalmente en el establecimiento de estándares mínimos de la oferta asociados a.

a) criterios de calidad preferentemente para los oferentes no públicos

b) ofrecimiento directo de los servicios educativos en forma gratuita o subsidiada

c) creación de incentivos a la oferta privada especialmente de alta calidad

En general los estándares mínimos de funcionamiento y de oferta se estructuran sobre la base de la protección del interés público y del usuario de los servicios públicos. Ello al tiempo se ha analizado en América Latina asociado a un relativo pasaje desde un estado educador hacia un estado evaluador (RODRÍGUEZ OSTRIA, 2000). También se ha considerado que esta dinámica es resultado de la diferenciación institucional pública y de una expansión de la oferta privada que facilita el establecimiento de políticas sistémicas de aseguramiento de la calidad (LEMAITRE, 2005).

El establecimiento de un nivel mínimo de estándares obligatorios de la oferta, en condiciones de mercados competitivos, tiende a ser sistémico y a descansas en la búsqueda de la igualdad: en que los diferentes oferentes compitan y ofrezcan servicios bajo estándares mínimos comunes. Es una política pública que se asocia a un universalismo básico en la oferta. En la región sin embargo, por la autonomía de la primera reforma universitaria y la diferenciación privada de la segunda reforma, se ha conformado en general un modelo bimodal de regulación público - privado, en el cual no siempre los estándares son comunes. Estos además se 
han ido modificando, en general hacia mayores exigencias, a medida que la sociedad se democratiza, que aumenta su complejidad y sus demandas sociales, y que las propias dinámicas de la política pública aumenta su protagonismo fiscalizador (RAMA, 2006). Al tiempo, el aumento de estos estándares se constituye en uno de los determinantes de la lógica de la competencia entre los diversos oferentes, y que se retroalimenta directamente con los precios de las matrículas. Los estándares mínimos fungen en tal sentido tanto como una barrera de entrada a nuevos competidores anulando sus ingresos de tipo "polizonte", así como también inversamente como mecanismo que impulsa la salida de aquellos competidores que no tienen capacidad de asumir los nuevos costos y requisitos que imponen los estándares elevados.

La regulación mediante el establecimiento de indicadores mínimos de cumplimiento obligatorios, se asocia a la construcción de las competencias básicas que debe adquirir cada persona en términos de saber hacer y de su ciclo escolar respectivo. Ellas se caracterizan en general por ser mínimos curriculares, académicos institucionales, de infraestructuras o de aprendizajes esperados. Son regulaciones que más allá de sus características especificas, tienden a imponer determinados modelos educativos, al fijar las variables mínimas de docentes, infraestructura, currículos, cargas académicas, perfiles de egreso, tipos de evaluaciones, sedes de apoyo, relación de tutores por estudiantes, áreas en las cuales se puede ofertar, y de los aprendizajes. En esta lógica gubernamental del aseguramiento de la calidad, que se ha conformado desde mediados de los 90 en toda la región, marcada por la tendencia a la regulación, es que se soportan los diversos modelos educativos a distancia en la región con sus propias diferenciaciones y tipologías (RAMA, 2008).

La primera generación de políticas normativas en educación a distancia, estuvo marcada por las leyes de creación de las universidades a distancia en los años 70 y 80 en Costa Rica (UNED), Venezuela (UNA) y Colombia (UNAD). Posteriormente los marcos normativos de la educación a distancia se caracterizaron por imponer un tipo de modelo único semipresencial, a través de unos criterios mínimos de una oferta. 
Fueron políticas de regulación de la educación a distancia de segunda generación e impusieron criterios que no sólo eran mínimos, sino que establecían los límites de la libertad y las formas de funcionamiento de la modalidad. Eran criterios que limitaban algunas ofertas de carreras, imponían la existencia de centros de apoyo presenciales, determinaban un porcentaje de actividades presenciales y fijaban que los exámenes o defensas tenían que realizarse en forma presencial. Ello impuso un modelo unimodal a distancia de prestación del servicio educativo con limitaciones de oferta, y permitió la existencia de un sistema bimodal: presencial y a distancia (semipresencial).

La educación superior en América Latina, fue en su totalidad unimodal de tipo presencial, hasta muy recientemente. Recién a inicios de los años 80 como parte de un proceso tardío y exclusivamente en pocos países, tales como Colombia, Venezuela, Costa Rica y México, se comenzaron a introducir ofertas públicas de educación a distancia sobre la base fundamentalmente de la creación de instituciones orientadas a ello que definimos como de primera generación. Al tiempo fue parte de un proceso más amplio de diferenciación de modalidades de enseñanza y de la propia educación superior. Ello fue acompañado posteriormente en los años 90 por el ingreso de instituciones privadas orientadas a ofertar educación a distancia bajo modelos semipresenciales, así como de aumento de éstas ofertas por parte de instituciones tradicionales presenciales. En esos contextos se aprobaron normas de regulación de la educación a distancia en diversos países consolidando el modelo semipresencial. Finalmente desde los años 2000 con la digitalización se inicio un mayor crecimiento de estas ofertas semipresenciales mediante la inclusión de componentes virtuales con uso de plataformas tipo LMS, y que al tiempo dieron un mayor impulso a la oferta privada. Ello abrió una transición, bastante generaliza$\mathrm{da}$, desde ofertas semipresenciales a semivirtuales, pero al tiempo en ese proceso de transición se impulso el inicio de ofertas 100\% virtuales con profesores interactuando en plataformas y no presencialmente, fundamentalmente en la segunda década del siglo XXI como analizaremos a partir de seis casos. Ello comenzó a impulsar un cambio en la regulación y 
una presión para la habilitación de esta modalidad en la oferta a distancia. En México y Colombia donde se produjeron antecedente de estas políticas de habilitación de ofertas virtuales, el RVOE (Registro de Validez Oficial de Estudios) en México y el Registro Calificado en Colombia, establecen las características que deben tener los programas para ser autorizados en las distintas modalidades, y habilitan diversas tipologías o modelos de educación a distancia con sus propios estándares diferenciados apara el licenciamiento de programas.

Este desarrollo ha llevado a que para el momento actual la educación a distancia esté presente con diversa intensidad en toda la región. Al tiempo, en cada vez más países la oferta a distancia se expresa a través de multimodalidades, gracias a nuevos marcos normativos que permiten ofertas virtuales y con ello el pasaje desde un esquema bimodal (presencial y semipresencial a distancia), hacia dinámicas multimodales en el cual se incluyen diversidad de combinatorias entre ofertas virtuales y presenciales y entre ellas ofertas $100 \%$ virtuales sin actividades presenciales e incluso tipo MOOCs (cursos masivos abiertos en línea) sin tutores al interior de las ofertas a distancia.

\section{Análisis de las nuevas normativas de la educación a distancia en América Latina}

En los últimos años está irrumpiendo un conjunto de nuevas regulaciones de la educación a distancia, que definimos sobre la tercera generación de las regulaciones en este sector, que está habilitando ofertas $100 \%$ virtuales con mayores niveles de flexibilidad. Es este un resultado del avance de la programación informática y de las tecnologías de comunicación e información digitales, pero también se asocian a nuevos paradigmas conceptuales sobre la expansión de esta modalidad y de menores niveles de resistencia. También se asocian a la búsqueda de lograr mayores niveles de cobertura y a un aumento de la demanda por este tipo de estudio. Finalmente es de destacar mayores niveles de expansión y 
conectividad de los accesos a las redes digitales. Esta tercera generación de políticas de regulación de la educación a distancia en educación superior habilitan diversidad de tipos de ofertas, y conforman un realidad de la oferta en base a "multimodalidades" por parte de los sistemas de educación superior locales. Representan un aumento de la diferenciación de los sistemas de educación superior y específicamente de la educación a distancia. Sin embargo, una mirada más profunda, nos permite verificar la diversidad de éstas distintas regulaciones nacionales, su dispersión y dificultad de convergencia, su entonación diferenciada, así como sus particularismos, más allá que ellas habiliten ofertas de educación superior totalmente virtuales.

Esta nueva normatividad representa una tercera generación de políticas de regulación de la educación no presencial o a distancia, y que al tiempo permiten una mayor diferenciación institucional en sus respectivos sistemas, atento a sus sutilezas y características.

Realizaremos a continuación un análisis comparativo de la situación de la educación a distancia en diversos países, y específicamente de la evaluación de las nuevas normativas de educación superior a distancia en Argentina, Brasil, Ecuador, Perú, Paraguay y El Salvador, que han sido algunos de los países con nuevas normas.

\section{La educación a distancia y su regulación en Ecuador}

En Ecuador, la educación a distancia tiene un largo recorrido, fundamentalmente por el aporte de la Universidad Técnica Particular de Loja (UTPL), expandida bajo un modelo semipresencial de primera generación (CORREA, 2013). La incidencia de la matricula bajo la modalidad a distancia se distribuye entre el posgrado y el grado, y representaba el $11.34 \%$ de la matrícula superior en el país para el 2013. En la cobertura de educación a distancia, el sector privado es altamente dominante: mientras el sector público para el 2013 cubría el 10,85\% de la matricula, el sector privado cubría el restante $89,15 \%$. Tal situación muestra una 
alta concentración institucional, tanto en el grado como en el posgrado. Para el 2012, la matricula de posgrado a distancia era de 5947 estudiantes y se concentraba en la Universidad Técnica Particular de Loja (UTPL) el $86,7 \%$ de los estudiantes, en tanto que la matrícula de Universidad de Especialidades Espíritu Santo (UEES) alcanzaba al 4,18\% y en UNIANDES era el 9,08\%. La concentración de la UTPL en el mercado a distancia a nivel del grado, era el 74,76\% de la matricula a distancia para ese año.

Impulsando un cambio del modelo unimodal a distancia, la Ley Orgánica de Educación Superior (LOES) del año 2010, estableció la existencia de las multimodalidades, al disponer en el artículo 169, que el Consejo de Educación Superior (CES) deberá aprobar el reglamento de "De régimen académico y títulos, y de régimen de posgrado; y de las modalidades de estudios: presencial, semipresencial, a distancia, en línea y otros". (SENESCYT, 2010). Ello fue un cambio en tanto la norma anterior del Consejo Nacional de Educación Superior (CONESUP) solo autorizaba ofertas presenciales, semipresenciales y a distancia (RUBIO y MOROCHO, 2014). La nueva LOES, además estableció claramente a través del artículo 122, la diferenciación en el otorgamiento de los Títulos, al disponer que en éstos se deberá "establecer la modalidad de los estudios realizados”. En esa línea, posteriormente en el 2013, el Reglamento de Régimen Académico (RRA) aprobado por el nuevo órgano de regulación, el Consejo de Educación Superior (CES) estableció en su artículo 39 un carácter más amplio al disponer que las instituciones de educación superior podrán impartir sus carreras y programas bajo las modalidades de estudios o aprendizaje presencial; semipresencial; dual; en línea; y, a distancia.

Finalmente en el 2015, el CES aprobó un reglamento específico de educación a distancia, de acuerdo a lo dispuesto por la Ley Orgánica de Educación Superior (2010) y el Reglamento de Régimen Académico (2013). Este, continúa las líneas trazadas por los marcos normativos referidos, y en tal sentido modifica la norma anterior de educación a distancia del año 2009 de cuando existía el CONESUP y que sólo habilitaba educación a distancia tradicional. 
La nueva norma establece en los aspectos de gobernanza del sistema, la habilitación de diversas modalidades sobre parámetros comunes y a la vez diferenciados de aseguramiento de la calidad. Ella establece mínimos niveles de funcionamiento para la modalidad así como para las distintas tipologías (a distancia y virtual). Dispuso la existencia de una unidad administrativa y académica de gestión en un nivel elevado de la estructura institucional y que tanto la gestión académica como tecnológica estén a cargo de unidades especializadas, con personal especialmente formado en la modalidad, y conformado el equipo académico en forma articulada entre profesores, profesores instructores, tutores y técnicos, y donde el profesor es el responsable académico del curso.

Se establece que la oferta de educación a distancia se debe realizar con apoyos tutoriales locales a través de centros de apoyo y actividades presenciales, en tanto que la oferta de educación en línea, se debe apoyar en recursos de aprendizaje digitales e interactivos y prácticas pre profesionales presenciales, a cargo de tutores especiales. La norma establece una cantidad de tutores, profesores y estudiantes por curso, siendo en el caso de tutores uno por cada 50 estudiantes adicionando un monto asociado a la tasa asociada a la deserción existente. Sin embargo, la norma incluye la posibilidad de utilizar MOOCs hasta el $15 \%$ de los cursos, sujetos a evaluaciones presenciales. Es esta la primera norma de la región que incorpora la modalidad de educación automatizada de los cursos en línea. La norma habilita la posibilidad de periodos académicos flexibles con tiempos diversos de estudios pero con un mínimo de 8 semanas por curso y propende a la articulación de las asignaturas entre las diversas modalidades para facilitar trayectorias continuas entre ellas. Se dispone que los estudios de grado requieran la existencia de exámenes finales de conocimiento o de habilitación profesional

En relación a las estrategias de aprendizaje, se establece la necesidad de mecanismos de acceso flexibles y, en el caso del sector público, nivelación a cargo de las instituciones para los estudiantes de menos formación, a cargo de una unidad curricular de nivelación donde se documente las formas de evaluación del ingreso y los cursos de nivelación y 
de formación de capacidades en auto aprendizaje y en el aprendizaje bajo estas modalidades. Estos cursos propedéuticos de nivelación pudieran ser continuos durante los recorridos académicos para garantizar los aprendizajes y no sólo al inicio.

Los Centros de Apoyo requeridos para ofertar bajo la modalidad de educación a distancia, deben ser autorizados por separado a los programas. La norma dispone finalmente que no se pueda ofertar carreras o programas en línea o a distancia, en Ciencias biológicas y afines, Medio ambiente, Ciencias físicas, Ingeniería y profesiones afines, Industria y producción, Arquitectura y construcción, Agricultura, Silvicultura, Pesca, Veterinaria y Salud (RRA). Ello está además en concordancia con la estratificación que se ha realizado en el sistema por parte del CEAACES y en tal sentido se dispone que las carreras de licenciatura y posgrado de especialización y maestría podrán ser ofrecidas en línea por las IES que han tenido Categoría A y B, en una o dos de las últimas dos evaluaciones.

\section{La educación a distancia y su regulación en Argentina}

La educación a distancia tiene un largo desarrollo en Argentina y la Red Rueda que nuclea a las Universidades e Instituciones Universitarias con programas de educación a distancia se formó desde el año 1990. Estudios realizados muestran el continuo avance de instituciones con ofertas de educación a distancia. En 1993 eran apenas 6 universidades, 2 públicas y 4 privadas, que eran el $8 \%$ de las instituciones universitarias existentes ofrecían educación a distancia. En el 2000 eran 25 instituciones que para ese momento representaban el $27 \%$ de las instituciones las que ofrecían educación a distancia. En el 2007 pasaron a ser 48 instituciones que representaban el 46,6\% de las instituciones. Para el 2006 la matrícula a distancia de grado alcanzó a 40.495 estudiantes que representaban el $4,8 \%$ de la matricula total de grado de ese momento. En ese año la oferta a privada a distancia de 20.786 estudiantes superó levemente a la oferta a distancia del sector público que fue de 19.709 estudiantes. Desde ese 
momento la oferta de educación a distancia se expandió en forma significativa, y especialmente la oferta del sector privado. Gracias aún al mayor peso de este sector en la oferta a distancia, la incidencia del sector privado en la educación superior pasó del 20 al 25\% de la cobertura. ${ }^{1}$ Para el 2015, había 540 carreras a distancia, de las cuales el $51 \%$ era del sector público, correspondientes a $22 \%$ en el posgrado, $47 \%$ a grado y el restante en pregrado. En el posgrado, el sector privado tiene mayor peso en la oferta de diplomados (90\%), y menor a medida que aumenta los niveles: en las especializaciones $32 \%$ y $25 \%$ en las maestrías. Las ofertas por su parte están altamente limitadas a unos campos disciplinarios ya que las ciencias sociales y humanas con el $43 \%$ de los programas y las ciencias económicas con el 37\% acaparan la mayoría de la cobertura (RUEDA, 2016).

Es de destacar en este proceso que si bien son muchas las instituciones con ofertas de programas a distancia, son unas pocas instituciones las que cubren la mayoría de la oferta como resultado de procesos de especialización, aseguramiento de la calidad y diferenciación regional. Destacan especialmente en el sector privado las Universidades Siglo XXI con una matrícula con más de 45 mil estudiantes a distancia ampliamente extendida en todo el territorio nacional, la Universidad Católica de Salta, la Universidad Blas Pascal. Todas son instituciones expandidas desde el interior del país

El país no tiene datos estadísticos oficiales de educación superior a distancia, lo cual hace complejo su análisis. El análisis se concentra en la matrícula de las instituciones de educación con fuerte oferta a distancia y en datos dispersos de algunas instituciones. Ello permite suponer que para el momento actual, la oferta a distancia, tanto pública como privada, pudiera considerarse que está cerca del $5 \%$ de la matrícula, o sea en el entorno de los 100 mil estudiantes, donde el sector privado cubre la mayoría de la matricula.

1 Diario Clarín 2/6/2013. "La educación a distancia, a paso lento entre los universitarios". https:// www.clarin.com/economia/economia/educacion-distancia-paso-lento-universitarios_0_ BkgbScDovXI.html 


\section{Primera generación de políticas de regulación en educación a distancia}

La estructura legal de soporte de la educación a distancia se apoya en la Ley Federal de Educación No 24.195 del año 1993, cuyo artículo 24, expresa que "la organización y autorización de Universidades alternativas, experimentales, de postgrado, abiertas, a distancia, institutos universitarios tecnológicos, pedagógicos y otros creados libremente por iniciativa comunitaria, se regirán por una ley específica." Ello derivó en la Ley Educación Superior N ${ }^{\circ} 24.521$ del año 1995, que autorizó la creación y el funcionamiento de las diversas modalidades a distancia, previa evaluación de su factibilidad y de la calidad de su oferta académica, y sujeto a la reglamentación del Poder Ejecutivo.

A partir de allí, el marco de funcionamiento de la educación a distancia se realizó por normas inferiores, al tener los gobiernos esta facultad de regulación. Ello se dio por el Decreto 81 de 1998 y la Resolución Ministerial No 1717 del año 2004. Ellas fueron de tipo restrictivo a su expansión (SANTÁNGELO, 2008). En el 2011 se aprobó la Resolución Nº 160 , de regulación de todos los posgrados, que establece los criterios específicos para la oferta a distancia en posgrado.

La Resolución del año 2004, estructura un sistema a distancia de tipo semipresencial al obligar a la existencia de Centros de Apoyo para la oferta del servicio en esta modalidad. Esta Resolución profundiza en los requisitos del 1998, que fue la primera que se aprobó por el Ministerio de Educación, de acuerdo a las disposiciones y restricciones existentes en la Ley de Educación Superior 24.521 del año 1995 y sus decretos reglamentarios. La normativa de educación a distancia establece los requisitos y características que debe tener las ofertas universitarias para obtener el reconocimiento oficial y la validez nacional de sus títulos y ofertas.

Esta Resolución Ministerial No 1717, se basa en una jerarquía de requisitos asociados a los niveles de enseñanza, con un mayor nivel de regulación en los posgrados. Asume que a un mayor nivel de formación se requieren mayores estándares y controles de la oferta. Así, se estableció una escala de exigencias desde el primer nivel de Doctorado, 
Maestrías y Especialidades, un segundo nivel dado para las carreras de grado como Licenciaturas, Ingenierías y demás títulos mayores, y un tercer nivel más flexible dado por las carreras universitarias menores tales como las tecnicaturas y títulos intermedios, que en Argentina se denominan pregrados. Se establece que a mayor nivel de formación ofrecida, se demanden mayores exigencias en los componentes principales para autorizar la oferta a distancia.

Los ejes de la regulación y funcionamiento de la oferta a distancia, fueron la explicitación documental y práctica de la existencia de un modelo educativo, una efectiva dinámica de interacción docentes / estudiantes y estudiantes entre sí; materiales especiales para la enseñanza; requisitos mínimos de niveles de formación docentes; tecnologías digitales de información y comunicación; un formato de evaluación y el funcionamiento de sedes de apoyo. Ello conformó en síntesis un modelo de educación a distancia con apoyo de sedes y plataformas, sujeto a autorizaciones centrales del Gobierno altamente documentales de insumos y procesos. Bajo este modelo se expandió una oferta de pregrado y en menor proporción posgrados con apoyo de sedes. Los centros de apoyo tenían requisitos mínimos de funcionamiento, podían ser establecidos mediante acuerdos con instituciones educativas y debían ser autorizados por la Secretaría de Políticas Universitarias, para las diferentes actividades presenciales o mediadas tecnológicamente, de las carreras o programas bajo la modalidad de Educación a Distancia.

En el 2007, se estableció un acuerdo entre el Ministerio de Educación Nacional, el Consejo Federal de Educación, la Secretaría General del Consejo Federal de Educación, la Comisión Federal de Registro y Evaluación Permanente de las Ofertas de Educación a Distancia, que estableció una gestión y administración federal de las ofertas formativas de educación a distancia posibilitando una articulación intra e interjurisdiccional para la gestión federal de los estudios a distancia. Ello permitió que las ofertas de educación a distancia - más allá de trámites y requisitos - tuvieran cobertura nacional, a diferencia de las ofertas presenciales que eran casi exclusivamente Provinciales. 
Ese escenario configuró un espacio potencial de crecimiento especial a las instituciones con ofertas a distancia.

El aseguramiento de la calidad de la educación a distancia es diferenciada a nivel del grado y del posgrado. En el grado para ofertarla se requiere que el programa deba obtener el reconocimiento oficial y la consecuente validez nacional por parte del Ministerio de Educación. Sin embargo, si la carrera ha sido declarada de interés público debe ser acreditada por la CONEAU para lo cual se evalúan diversos componentes, tales como carga horaria, contenidos curriculares e intensidad de la práctica prevista en la carrera, de acuerdo a diversos estándares de calidad propuestos por el Consejo Interuniversitario Nacional (CIN) y aprobados por el Ministerio. En el área de posgrado, para todas las ofertas de especialización, maestría y doctorado, la acreditación por parte de la CONEAU es obligatoria. En estos casos, y recién en segunda instancia, el Ministerio de Educación otorga reconocimiento sujeto a si el programa fue acreditado.

El haber establecido la acreditación obligatoria para los tres niveles de posgrado y para las carreras de interés social que a la fecha son 13, ha llevado a una diferenciación de la acreditación como proceso obligatorio, de una nueva figura llamada categorización, que es voluntaria en el marco de la presentación a la acreditación. En este sentido, la acreditación refiere a los estándares mínimos en tanto que la categorización refiere a estándares superiores de calidad. Ésta en base a determinados estándares, asigna las letras A, B o C, según el perfil de calidad del programa. Se incluyen aspectos académicos, como aspectos organizacionales e institucionales. La vigencia de los procesos de acreditación es de tres o seis años, siendo la primera vez que se presenta una carrera ante CONEAU de un máximo de tres años.

Las políticas de acreditación de CONEAU han ayudado a detener la aparición de instituciones extranjeras y programas académicos externos que ofrezcan directamente o a través de convenios con universidades locales. Es en este sentido una educación a distancia local. 


\section{Tercera generación de políticas de regulación en educación a distancia}

La regulación inicial, tuvo su primer cambio con su flexibilización en el área de posgrado, con la aprobación de la Resolución N No 160 del 29 de diciembre del 2011 que estableció nuevos ejes de la oferta de posgrados en el país, al cambiar la norma anterior de regulación de posgrados dada por la Resolución 1168 de 1997 imponiendo también nuevos criterios para los posgrados a distancia. Esta norma, se aprobó como derivación de recomendaciones del Consejo Interuniversitario Nacional (CIN), y mantiene la acreditación obligatoria por parte del CONEAU para todas las instituciones de las ofertas de posgrado, en los niveles de Especialización, Maestría (de todas, distinguiendo además las maestrías académicas de las de investigación) y de doctorado. Otros tipos de posgrado que den lugar a otras certificaciones no están regulados.

La nueva normativa de posgrado del 2011 introdujo una mayor flexibilidad y nuevas oportunidades para la educación a distancia en este nivel, al permitir una oferta totalmente virtual. La norma establece que los posgrados pudieran ser tanto presenciales como a distancia, y mantiene el criterio de la norma del 2004 que engloba en esta categoría todas las propuestas educativas tales como educación virtual, elearning, semipresencial, asistidas, blended learning, etc. Sin embargo, la nueva norma no establece la obligatoriedad de sedes para realizar las ofertas a distancia. Con este escenario, el sistema a nivel de posgrado pasó a tener una mayor flexibilidad que el grado al habilitar todas las ofertas de posgrados a distancia, incluyendo la oferta virtual. Ello está habilitado en todos los niveles regulados de la oferta de posgrado, sujeto a la aprobación por parte de CONEAU pero no a la norma explícitamente.

Este marco normativo de posgrado otorga más flexibilidad a la oferta de posgrados, en tanto determina tres tipos de posgrado: estructurados, semi estructurados y personalizados. Tal flexibilidad tiene algunas limitaciones, en tanto se dispone que la habilitación de la oferta a distancia sea para los cursos, habiendo excepción para las prácticas que hubiera 
en dichas carreras, y a la obligatoriedad de presencialidad en el momento de defensa de presentación de las tesis cuando corresponde. Se establece que tanto las maestrías como los doctorados deberán tener una defensa oral y pública en una sede física de la institución universitaria, preferentemente donde la carrera fuera dictada.

La regulación de los procesos de oferta a distancia dispone la obligatoriedad de reglamentos, plataforma, formas en que se proponga la evaluación, el carácter presencial de la práctica cuando se incluya en el programa académico y las características de las unidades de apoyo (tecnológica y académica) cuando así se prevean. Se dispone que se deban presentar totalmente los recursos de aprendizaje de la carrera que requieran los estudiantes durante el primer semestre, en el momento de la solicitud de autorización de oferta.

Un nuevo cambio se produjo en el 2017 mediante la Resolución No 2641, se modificó la normativa de regulación de la educación a distancia en el nivel superior del año 2004 en los aspectos específicos de la oferta de grado y pregrado. La nueva normativa fue resultado de un amplio consenso y discusión entre la Secretaria de Políticas Universitarias, el Consejo Nacional de Universidades y la agencia de acreditación CONEAU.

El eje de la nueva norma es una mayor flexibilización con aumento de las diversidades de tipologías en la oferta de educación a distancia, incorporando la oferta virtual que pasa a habilitarse para el grado sujeta a diversos criterios. Entre ellos se establece que la educación a distancia, sea semipresencial o virtual, siempre tendrá un profesor designado que dicte clases o realice seguimiento de los docentes auxiliares (tutores).

La norma dispone el fin de un modelo radicalmente separado de presencial y a distancia, disponiendo que en las carreras presenciales hasta el 50\% de la carga horaria se pueda realizar mediante su dictado a distancia. En esta línea, dispone que cuando entre el $30 \%$ y el $50 \%$ sea realizado a distancia en la oferta presencial, ella deba someter a evaluación su sistema de educación a distancia. También en los casos de carreras presenciales intensivas se deberán hacer explícitos los modelos de apoyo a distancia. 
La educación no presencial, se define cuando la oferta presencial sea menos del $50 \%$ y bajo la modalidad a distancia, se puede ofertar desde pregrado a posgrado.

El sistema argentino, constituye un modelo de regulación donde el licenciamiento y la acreditación se han articulado conjuntamente a cargo de la CONEAU, creando complejas funciones entre ambos niveles. Actualmente se ha establecido una categorización donde el primer nivel de la acreditación (A) es obligatorio, y donde las categorizaciones de mayor calidad $\mathrm{B}$ y $\mathrm{C}$ son optativas, a su vez dependiendo de si son carreras de riesgo social tienen un nivel de obligatoriedad en el nivel mínimo.

Se establece que la educación a distancia tendrá los mismos estándares de evaluación que la educación presencial y que los sistemas de educación a distancia de las instituciones del país deberán pasar por procesos de evaluación. En tal sentido, se avanza hacia una evaluación obligatoria además de sus procesos internos de gestión de la educación a distancia en los niveles mínimos de estándares requeridos que se establecen. La normativa no obliga a la existencia de unidades de apoyo, que se diferencian entre aquellas para las actividades académicas y aquellas para las actividades tecnológicas. Se establece que las de actividades académicas serán cuando hay clases presenciales, tutoriales, prácticas o evaluaciones en ellas. Podrán existir también unidades de apoyo mixtas. Las unidades de apoyo pueden ser figuras jurídicas externas, permitiendo por ende la terciarización o franquicia.

La reglamentación establece que serán obligatorias las prácticas presenciales cuando sean carreras de grado de interés social, y que además lo establezcan los estándares de acreditación. Es en este sentido es una regulación con alta variabilidad dada a lo que resuelva la agencia de acreditación CONEAU. 


\section{La educación a distancia y su regulación en Perú}

Los datos de la educación a distancia en Perú son escasos y poco actualizados. Sin embargo, para el 2010 investigaciones, registraban una matrícula de educación a distancia, en sus diversas modalidades, en el Perú de 60.882 estudiantes que representaban el 7\% de la matrícula. Esta incidencia es inferior a los indicadores de la región, y corresponde a un escenario cuantitativo de países con escasa regulación. La participación de la matrícula a distancia en el país se distribuía en 35.089 estudiantes en la modalidad semipresencial y 24.793 en la modalidad no presencial, que representaban respectivamente el $4 \%$ y el 3\% de la educación superior. Ello mostraba la existencia de multimodalidades. Tal oferta a distancia estaba concentrada en una cantidad pequeña de las 137 universidades existentes. Fundamentalmente se localizaba en algunas de las 86 universidades privadas para ese momento, a nivel del grado. En el sector privado, a nivel del grado, el 10\% de los estudiantes, realizaba sus estudios a distancia o semipresencial. A diferencia en el sector público, también a nivel del grado, con 51 universidades, la matricula a distancia y semipresencial, sólo alcanzan al $4 \%$ del total de sus estudiantes.

En el sector de posgrado, la matricula semipresencial y a distancia alcanzó a 9.790 estudiantes y representaba el 17\% de los estudiantes de este nivel. El 14\% o sea 8.062 estudiantes estaban bajo oferta semipresenciales y el $3 \%$ restante en oferta a distancia. A diferencia el sector público sólo tiene el $7 \%$ de sus estudiantes bajo estas modalidades, por lo que es en el sector privado y en el nivel de posgrado donde se han expandido los modelos semipresenciales y a distancia en el país.

Este modelo a distancia semipresencial o llamado blended learning en Perú también - se ha expresado en una red de centros de apoyo o filiales para apoyar las actividades. De muy irregular composición estas sedes han sido un foco de fuerte crítica por parte de los organismos públicos que han buscado limitar su crecimiento. 
La Ley de Educación Superior de 1983 no había habilitado la existencia de ofertas de educación superior a distancia, pero al amparo de la autonomía de las Universidades y de la Ley General de Educación de 2003 ( No 28.044) que definió esta modalidad, año a año han ido aumentando el número de universidades, la cantidad de programas a distancia y la matrícula, en el marco de una confusa legislación ya que no se podían autorizar programas a distancia, al no existir un basamento de la ley universitario (RODRÍGUEZ, 2012).

Esta ausencia de un marco de regulación e incentivo a la educación a distancia en el Perú, ha determinado que esta modalidad haya tenido una expansión marginal, comparativamente con los restantes países de la región, pero al tiempo carente de claros estándares de regulación, en tanto entraban sus ofertas dominantemente en el marco de la autonomía de las universidades. La existencia de su baja oferta se verifica por ejemplo en la alta cobertura en la capital y la escasa cobertura del interior donde debiera haber tenido más oferta. A pesar de que Lima contaba con aproximadamente el $27 \%$ de la población del Perú, cifras del INEI señalan que para el 2012 el 41.7\% de la población de estudiantes de pregrado estudia en la capital. La concentración allí era elevada, al tener la capital el 39\% de las universidades existentes.

La educación superior en Perú inició un nuevo ciclo como resultado de un cambio de su marco legal con la aprobación de nuevas leyes de regulación, dadas por la Ley Universitaria No 30.220 del año 2014 y la Ley de Institutos y Escuelas de Educación Superior No 29.394 del 2015. Estas reconfiguran el sistema de educación superior en el país y cierran el largo ciclo iniciado de apertura que facilitó la diferenciación dado por la Ley de Universidades No 27.733 de 1983, y apoyado además por diversas normas posteriores que conformaron una dinámica universitaria centrada en la autonomía pública, la libertad de mercado privado, la autoregulación sistémica entre las Universidades públicas y privadas a través de la Asamblea Nacional de Rectores (ANR), un escaso financiamiento público y una prevalencia de lógicas de mercado, incluyendo ofertas con fines de lucro. 
La aprobación de este nuevo marco de regulación fue un proceso lento de avance desde lo que hemos llamado la segunda a la tercera fase de la educación superior en América Latina, y que abre un ciclo más centrado en la regulación y que propende a un mayor control de la oferta (RAMA, 2006). Ella se inició muy tibiamente con la Ley de creación del Sistema Nacional de Evaluación, Acreditación y Certificación de la Calidad Educativa (SINEACE) en el 2006 que asumió la potestad de autorizar y evaluar la creación y funcionamiento de filiales universitarias a través del Consejo de Evaluación, Acreditación y Certificación de la Calidad de la Educación Superior Universitaria (CONEAU). Sin embargo, su carácter voluntario, la continuación de creación de universidades, programas, sedes y filiales no se detuvo. También la Ley de SINEACE tuvo una alta lentitud de instrumentación, lo que llevo finalmente a que en diciembre del 2012, se aprobara una Ley de Moratoria, que impidió la creación de nuevas universidades y filiales por un periodo de cinco años.

De hecho se había expandido una educación semipresencial con apoyo de sedes y filiales en el interior con un relativo y escaso apoyo en recursos de aprendizajes y plataformas pero sin la aprobación de un marco de regulación que correspondiera a la primera generación de regulación de la educación a distancia. Hubo varias iniciativas de la Asamblea Nacional de Rectores (ANR) que inclusive presentó un anteproyecto de Ley al parlamento, pero ello no se produjo.

La nueva Ley Universitaria No 30.220 aprobada en el 2014, suprimió la Asamblea Nacional de Rectores y creó la Superintendencia Nacional de Educación Superior Universitaria (SUNEDU) como organismo público técnico especializado adscrito al Ministerio de Educación, con autonomía técnica, funcional, económica, presupuestal y administrativa, y con naturaleza jurídica de derecho público interno y pliego presupuestal. La Ley establece un marco de regulación altamente simplificado y restrictivo de la oferta de la educación a distancia, con un perfil homogéneo de la oferta universitaria, y con prohibiciones de oferta en los niveles de posgrado. En general, el enfoque de la Ley se apoya en un paradigma por el cual la calidad de la oferta universitaria se asocia a su carácter presencial, 
con escasa diferenciación de tipologías, limitante de la diversidad institucional y sin valorización de la innovación tecnológica y la incorporación de las tecnologías de comunicación e información en el funcionamiento de la oferta universitaria. Se podría sostener que este marco legal, corresponde a la primera generación de regulación de la educación a distancia vista regionalmente, pero que tiene su basamento en la inexistencia de regulaciones anteriores.

La Ley promueve un tipo de educación superior presencial, catedrática y de investigación. Los elementos referidos, destacan en relación al tratamiento dado a la educación a distancia, la innovación pedagógica, el uso de las tecnologías de comunicación a información y la diversidad de instituciones y dinámicas de las ofertas universitarias. En tal sentido la norma fue claramente restrictiva de esos componentes y estableció un marco normativo, que no permitirá un uso eficiente de los avances de las tecnologías de comunicación a información. Las normas no excluyen la educación a distancia, sino que establecen que la modalidad la puede definir la Universidad y que puede ser presencial, semipresencial y a distancia. Sin embargo, a la par, se define un modelo de regulación y de exigencias específicas para la educación a distancia que limita fuertemente la modalidad virtual e impone una modalidad semipresencial. En tal sentido, el artículo $\mathrm{N}^{\circ} 47$ de la nueva Ley, expresa que los estudios de pregrado de educación a distancia no pueden superar el 50\% de los créditos totales de la carrera bajo esta modalidad.

El artículo central de la regulación de la educación a distancia en la nueva ley establece:

Artículo 47. Educación a distancia Las universidades pueden desarrollar programas de educación a distancia, basados en entornos virtuales de aprendizaje. Los programas de educación a distancia deben tener los mismos estándares de calidad que las modalidades presenciales de formación. Para fines de homologación o revalidación en la modalidad de educación a distancia, los títulos o grados académicos otorgados por universidades o escuelas de educación superior extranjeras se rigen por lo dispuesto en la presente Ley. Los estudios de pregrado 
de educación a distancia no pueden superar el 50\% de créditos del total de la carrera bajo esta modalidad. Los estudios de maestría y doctorado no podrán ser dictados exclusivamente bajo esta modalidad. La SUNEDU autoriza la oferta educativa en esta modalidad para cada universidad cuando conduce a grado académico.

Aunque en la forma en la cual está redactado permitiría que algunos cursos puntuales pudieran ser $100 \%$ virtuales y otros $100 \%$ presenciales, si se cumpliese que en total se está por encima del $50 \%$ de presencialidad. Sin embargo, ella facilitará un modelo semipresencial del $50 \%$ como modalidad de oferta de la educación a distancia. Actualmente están en proceso de desaparición múltiples ofertas de acceso y las instituciones que están sometiendo sus cursos al licenciamiento por el SUNEDU de acuerdo a la nueva ley, muestra que los programas a distancia o semipresenciales, están perdiendo sus características, aumentando las horas de tutorías presenciales o de aulas presenciales para ajustarse a la norma del 50/50.

El impacto mayor de la nueva regulación es en el sector de posgrado, ya que la norma dispone que los estudios de maestría y de doctorado no pueden ser ofrecidos bajo la modalidad a distancia, sino que deben ser semipresenciales o presenciales. En tanta la ley define en el caso del nivel de maestría tanto las profesionales como las de investigación, en educación a distancia sólo puede ofrecerse a nivel de posgrado a los Diplomados, que es el primer escalón del posgrado y que corresponde a estudios cortos de perfeccionamiento profesional, en áreas específicas y para las cuales se debe completar un mínimo de veinticuatro (24) créditos.

Esta restricción, no resuelve la situación confusa de la normativa anterior, y limitará la expansión de la cobertura, así como restringirá la innovación pedagógica en la oferta universitaria, limitando el acceso fuera de las grandes ciudades. A estas limitaciones se agregan a otras de tipo tecnológicas y de conectividad para facilitar ofertas a distancia, ya que para el 2015, aunque los hogares como telefonía móvil eran el 79,7\% 
de los existentes, sin embargo los hogares con internet apenas representaban el 20,2\% de los hogares del país.

Hay además en el cuerpo normativo de la Ley de Universidades del 2014, una visión claramente negativa respecto a la educación a distancia. En tal sentido, en los artículos que establecen los requisitos para las diversas autoridades (del SUNEDU, (art 17) y para la dirección de las universidades, se dispone que el doctorado como exigencia académica deba ser presencial.

\section{La educación a distancia y su regulación en Brasil}

Brasil ha tenido una destacada normativa y regulación de la educación a distancia apoyada en el artículo 80 de la Ley 9394 del año 1996 (LDB) que habilitó la modalidad y en su reglamentación en el 2006 que estableció su marco de funcionamiento restrictivo. (Lupion at al, 2010). Ello correspondió a una primara fase caracterizada por una oferta semivirtual con apoyo de polos y con fuertes requisitos y que derivaron en una fuerte concentración de los proveedores. El modelo estaba altamente asociado a enfoques presencialistas. Sin embargo, tal normativa de la educación a distancia tuvo un cambio con la aprobación del Decreto 9057 del 26 de mayo del 2017. El decreto fue además complementado por la Portería No 11. La nueva norma, modifica fuertemente el marco de regulación anterior del año 2006 que había sido la base del fuerte proceso de expansión de la modalidad bajo un formato semipresencial y que había producido un crecimiento importante de la modalidad y con una concentración de la oferta en pocos grupos. La norma antecesora al nuevo Decreto 9057 había impuesto un sistema semipresencial basado en la existencia de polos de las universidades con altos requisitos para su autorización y lentos en la tramitación burocrática al estar sujetos a evaluación in situ por pares.

La nueva norma, permitió la inclusión de la oferta $100 \%$ virtual, pasando de una exclusiva modalidad a un formato multimodal que abre un nuevo escenario en el país. La norma legaliza la existencia de polos 
fuera del país, que actualmente mostraba apenas unos pocos. La norma no sólo se constriñe a la oferta en la educación superior, sino que habilita la oferta de educación a distancia en otros niveles sujetas a las autorizaciones de esos ámbitos, especialmente a las personas impedidas por salud de movilizarse, radicar en el exterior, estar privado de libertad o estar en regiones sin la existencia de ofertas presenciales.

La norma dispone la autorización de ofertas a distancia en la formación de grado y en posgrados latu senso, dejando a resolución de CAPES la autorización de ofertas de educación a distancia en el nivel de maestría y doctorado.

En los procesos de administrativos de autorización, la norma dispone que sólo se evaluaran las sedes a través de diversos parámetros y no individualmente a cada centro de apoyo. Además, la norma autoriza la realización de actividades académicas, no sólo en las sedes y centros de apoyo, sino también en ambientes profesionales. Facilita que las actividades de los polos se puedan articular entre varias instituciones mediante acuerdos.

El marco conceptual de la nueva norma es asociar la libertad de creación de polos a la calidad de las instituciones a través del concepto institucional de estas. Cuando mayor, más libertad tienen de creación de centros de apoyo, los cuales a su vez pueden ser propios o ajenos, pero bajo acuerdos de responsabilidad. La nueva norma amplia las modalidades, flexibiliza los controles, asocia la oferta a la calidad de la institución, donde a más calidad más libertad de ofrecer servicios sin mecanismos de control previo y además pone foco en los resultados y los compromisos de las instituciones.

\section{La educación a distancia y su regulación en El Salvador}

La oferta de educación a distancia en el país es escasa dadas las dimensiones del país, la alta cantidad de instituciones existentes en el sector privado que a su vez tienen una importante regionalización, 
favorecido por el alto porcentaje de población rural. El eje central de la cobertura de la educación superior a distancia descansa en la alta emigración poblacional. Ello ha promovido una oferta a distancia transfronteriza virtual correspondiente que correspondería a una cuarta generación de regulaciones de la educación a distancia. Actualmente menos del 1\% de la matrícula es a distancia, y en su mayoría personas fuera del país.

En el año 2012 a través del Decreto No 19 se aprobó el Reglamento especial de la educación no presencial en el Salvador por parte del Ministerio de Educación. Aunque es esta la primera norma aprobada en el país en la materia, por su contemporaneidad y por incorporar la educación $100 \%$ virtual, se puede considerar como una norma de la tercera generación de la educación a distancia. El eje hilvanador de la norma es el carácter no presencial de la educación, y la articulación entre ambas modalidades. Ella define dos dimensiones: la relación no presencial sin obligación de presencia física y aquella con presencia. La diferenciación está dada por la ausencia total o parcial de los estudiantes, docentes o tutores. En tal sentido se diferencia entre modalidad no presencial y modalidad mixta-semipresencial. Sin embargo, la norma limita la oferta de tipo MOOCs, que además para entonces no existía.

La norma habilita el tránsito entre ambas modalidades, pero limita que no se puedan cursar a la vez. Sin embargo establece que los programas de educación a distancia deben tener equivalencias con las ofertas presenciales con un sistema preestablecido de equivalencias. Igualmente dispone que se puedan realizar equivalencias con pruebas. La norma autoriza las ofertas tanto a nivel del grado como del posgrado, y establece que los cursos deben ser anuales o semestrales con un mínimo de 16 semanas para cada curso. En tal sentido, la norma no habilita la confluencia de intensidad de enseñanza y distancia.

Las instituciones deben garantizar que las personas que ingresan al campus virtual sean las personas que corresponde, pero se dispone que la evaluación puede ser virtual y sólo en caso de ser necesaria ser presencial.

La norma dispone que la asesoría, la tutoría y el diseño incluso puedan ser realizadas por la misma persona, permitiendo modelos no 
presenciales menos complejos, probablemente de acuerdo a la dimensión del mercado. Se dispone al tiempo un máximo de un tutor por cada dos grupos de 15 personas en cada programa.

\section{La educación a distancia y la regulación en Paraguay}

Paraguay aprobó la Ley de Educación Superior, No 4995 en el año 2013 que favoreció la habilitación de la educación a distancia. Ello condujo a la resolución del Consejo de Educación Superior (CONES) n 63 de febrero del 2016, por la cual se aprobó el Reglamento de la Educación Superior a Distancia y Semipresencial.

La norma dispone que los programas a distancia puedan ofrecerse en instituciones legalmente habilitadas que dispongan de infraestructura, equipamientos y profesores capacitados, y que sus programas, de pregrado, grado y posgrado, incluyendo doctorado, sean autorizados, diferenciando entre una modalidad elearning (virtual) y una blearning (semipresencial). Sin embargo dispone que al menos un examen debe ser presencial, aun cuando habilita que en casis especiales puede ser a distancia. La norma dispone la existencia de instituciones a distancia considerando sus especificidades, requiriéndose docencia, investigación y extensión. Concibe un equipo de trabajo y diferencia tareas entre docentes especialistas, tutores o asesores, especialista en diseño instruccional y soporte tecnológico.

La norma no regula las características de los centros de apoyo, y promueve ofertas interuniversitarias, e incluso alianzas entre instituciones extranjeras y nacionales.

No se diferencian los títulos entre las distintas modalidades, y'se dispone que el CONES establecerá las áreas en las que se pueda implementar la modalidad a distancia. De acuerdo a la Ley de la agencia de acreditación, ANEAS, algunos programas deben estar acreditados para su oferta. 


\section{La educación a distancia y su regulación en Costa Rica}

El país tiene un sistema de regulación dual, por lo que existen dos normativas en general, siendo una pública por parte del CONARE y otra que impacta sobre el sistema de educación superior privada a cargo del CONESUP. La regulación sobre la educación privada en materia de educación a distancia, estuvo dada por el Decreto Ejecutivo $\mathrm{N}^{\circ} 35.810$ del 20 de enero de 2010.

En esta regulación sobre el sector privado se aceptan todas las propuestas tales como enseñanza semi-presencial, no presencial, abierta, educación en línea, aprendizaje electrónico (e-learning), aprendizaje combinado (b-learning), aprendizaje en red (networklearning), aprendizaje o comunicación mediada por computadora (CMC), cibereducación, teleformación y otras. Se dispone que las instituciones de educación superior deben disponer de una organización académica de gestión, seguimiento y evaluación específica para la modalidad. Debe haber programa de capacitación docente en las tecnologías y las metodologías pertinentes a esta modalidad.

Se dispone que si se tiene aprobada una carrera presencial, sólo deber hacerse una Ampliación en los aspectos relacionados con la modalidad virtual. Para su aprobación el CONESUP dispuso que se requiera apara ofertas por parte de las universidades, la existencia de a) Organización virtual. b) Infraestructura y equipamiento. c) Soporte tecnológico y funcionalidad. d) Disponibilidad y plan de producción de recursos de mediación educativa. e) Estimación cuantitativa de los tiempos de las actividades. f) Normativas de interés para los estudiantes. g) Perfiles de los docentes y sus responsabilidades académicas; incluyendo capacitación. h) Evaluación del aprendizaje, condiciones de seguridad y confiabilidad de las pruebas. Más allá de estos requisitos, existe una supervisión continua de la modalidad virtual. 


\section{Análisis comparativo de la regulación de la educación a distancia en los países seleccionados}

Los casos analizados se deben ver en primera instancia en el contexto de las evoluciones particulares de las dinámicas locales y las demandas de diferenciación y facilitación de las oportunidades de acceso para aumentar la cobertura. Pero al tiempo ellas muestran también una convergencia relativa asociado a la expansión de la innovación tecnológica y pedagógica que propende al uso de la educación virtual y facilita una transición de la educación a distancia tradicional, y por ende un impulso a la virtualización. Al tiempo ello permite en los diversos países la existencia de multimodalidades en la educación a distancia y aumenta la diferenciación institucional de la educación superior en general. En la norma de Paraguay por ejemplo, se habilita la existencia de instituciones a distancia. Es este un proceso general de aumento de la diversidad, más allá de la existencia de particularismos, como es el de Perú, que a diferencia se destaca en la aprobación contemporánea de políticas de educación a distancia que corresponden a la primera generación de éstas regulaciones.

La región se caracterizó por el desarrollo inicial de una oferta especializada de educación a distancia semipresencial, y en los últimos años se inició una nueva oleada de política pública que llamamos de tercera generación que habilita a las instituciones de educación superior a ofertar en forma también totalmente virtual. Ello introduce nuevas tipologías de modelos educativos a distancia, con compleja convivencia de políticas, tipologías, modelos y hasta culturas y formas de competencia institucionales. Esta dinámica puede ser vista como parte del propio proceso de diferenciación institucional (CLARK, 1991) o de innovación de las universidades (CLARK, 2000), y que en ambos casos permite el aumento de la cobertura.

La lógica bimodal - presencial y a distancia- y actualmente multimodal al agregar la educación virtual, marca el escenario de la regulación que tiende a establecer estándares de funcionamiento mínimos para las distintas modalidades. 
La existencia de multimodalidades en la oferta a distancia, es una derivación del pasaje desde las regulaciones exclusivas para la educación semipresencial a distancia típica de la 1 y 2 generación de la educación a distancia, hacia la habilitación de ofertas virtuales. De hecho las multimodalidades son resultado de la existencia de marcos normativos que han permitido la educación virtual en diversos países de la región, o de una conceptualización más flexible de la educación a distancia que habilite todas las modalidades a distancia, tanto semipresenciales como las exclusivamente virtuales

Aunque ello es resultado de que las normativas se han ido flexibilizando para ajustarse a nuevas realidades tecnológicas y demandas de mercado, y que al tiempo tornan a la regulación bajo tipologías más complejas, al no ser fácilmente diferenciables las distintas modalidades. La legislación argentina y la peruana siguen el camino de diferenciar en función de mínimos de presencialidad como parte de la cantidad de horas estándar de los cursos presenciales.

En general, las universidades e instituciones de educación superior, y especialmente las privadas, pero también desde el sector público, buscan ofertar bajo todas las modalidades en función de las oportunidades de las TIC, de las propias demandas y de los costos de los servicios. Así, es que acompañando esos diversos intereses es que se han tenido a ajustar los marcos normativos para habilitar la educación a distancia y actualmente la modalidad virtual. Ello crea una situación de oferta bajo multimodalidades, resultado de la flexibilización de los criterios de habilitación.

Con la habilitación de la educación virtual hay un lento y persistente corrimiento de la demanda estudiantil de educación a distancia a la modalidad virtual, lo cual facilita algunas resistencias institucionales a los cambios normativos. Representa un pasaje entre modalidades que se asocia a la conectividad, costo de los equipamientos, diversidad de ofertas, caída de los precios de las matrículas y desaparición de costos de traslado. Sin embargo, también se mantienen nichos de demandas educativas que se articulan más eficientemente a unas modalidades 
de educación a distancia que a otras, lo cual facilita el aumento de la cobertura a distancia en general.

Las normativas tienden a diferenciar con diversa intensidad los estándares de habilitación para las dos modalidades a distancia: en Argentina y Ecuador, están más detalladas, en tanto que las fronteras son más borrosas en El Salvador, Paraguay y Brasil. Ello también se da en las normativas virtuales anteriores de Colombia y México respectivamente. En Argentina, Perú y El Salvador, la educación a distancia propende a una orientación - aun cuando no exclusiva - hacia una articulación con la educación presencial, donde los estudiantes realizan asignaturas a distancia o virtuales, que se correlacionan o equivalen a las presenciales. Esta dinámica que no se constata fácilmente en las estadísticas, constituye una práctica creciente en muchas universidades. La mayor hibridación, articulación o movilidad, es uno de los ejes de las nuevas normas.

Así por ejemplo, en el caso de Ecuador, el Reglamento de Régimen Académico (RRA), del 2015, en su artículo 46 dispone que "Los estudiantes podrán tomar hasta un $15 \%$, en grado, y hasta un $20 \%$, en posgrado, de las asignaturas, cursos o sus equivalentes de la correspondiente carrera o programa en otras modalidades de aprendizaje, en tanto exista la oferta en la misma u otra institución de educación superior, siguiendo los procedimientos establecidos por cada IES, y siempre que la carrera o programa estén acreditados por el CEAACES en la misma o superior categoría" (CES, 2013). Promoviendo la multimodalidad, en el RRA adicionalmente se dispone que las IES puedan planificar el proceso de aprendizaje de una carrera o programa académico con este mismo o inferior porcentaje en otras modalidades de aprendizaje.

En las diversas normativas analizadas, no se establecen diferenciaciones en las certificaciones que aclaren que los estudios corresponden a una modalidad específica, salvo Ecuador, que establece que las certificaciones son diferenciadas y deben tener aclaratoria en el título de la modalidad específica en la cual fue aprobado por el Consejo de Educación Superior. 
La complejidad de crear diferenciaciones de las regulaciones para la diversidad de modalidades en ese continuo infinito casi entre lo presencial y lo virtual, parecería facilitar el establecimiento de sistemas de aseguramiento de la calidad que incorporen la medición de los resultados de los aprendizajes más allá de cuál sea la modalidad bajo la cual se adquirieron las competencias. Ello sin embargo sólo se aprecia en la normativa de Ecuador, donde la Ley Orgánica de Educación Superior (2010), dispone en su artículo 103 la creación de un examen nacional de evaluación de carreras y programas académicos al disponer que "Para efectos de evaluación se deberá establecer un examen para los estudiantes de último año de los programas o carreras. El examen será complementario a otros mecanismos de evaluación y medición de la calidad disponer". Además de ello, la evaluación de los aprendizajes cumple el rol de validar el propio licenciamiento del programa, en tanto la norma establece que "en el caso de que un porcentaje mayor del $60 \%$ de los estudiantes de un programa o carrera no logre aprobar el examen durante dos años consecutivos, el mencionado programa o carrera será automáticamente suprimidos por el Consejo Nacional de Evaluación, Acreditación y Aseguramiento de la Calidad...”. La norma dispone además que "en el caso de que se suprima una carrera o programa, la institución de educación superior no podrá abrir en el transcurso de 10 años nuevas promociones de estas carreras o programas".

En este caso, la flexibilización de los procesos de enseñanza a distancia con multimodalidades (semipresencial y virtual) parece correlacionarse con nuevos mecanismos de aseguramiento de la calidad no sólo en base a la evaluación de los insumos y procesos sino de los resultados de los aprendizajes, como eje para evaluar y validar a los propios programas educativos. Brasil lo incorpora en la determinación del concepto institucional, en el cual un componente de la definición está asociado a los resultados de los aprendizajes entre el ENEN y el ENADE y otro componente a la evaluación documental de insumos y procesos. En El Salvador, Costa Rica, Argentina, Perú y Paraguay ello está ausente. En Colombia y México, que tienen normativas de habilitación de la educación virtual anteriores, 
si están presentes en la existencia de los Exámenes de Estado del ICFES (Colombia) y en las pruebas de CENEVAL en México, en este caso incluso como mecanismo de egreso específico alternativo.

Las regulaciones de tercera generación de la educación a distancia, se caracterizan por la inclusión de la educación virtual. Sin embargo, se constata el inicio de referencias reguladoras sobre la educación automatizada (MOOCs), como en Ecuador donde se habilita a la realización cursos MOCCs en la malla curricular de los cursos virtuales sujetos a la realización de evaluaciones por las instituciones, sobre esos aprendizajes y competencias. En tal sentido, aunque la región está frente a una transformación normativa pasando de regulaciones que habilitaron formatos semi presenciales correspondiente a una etapa inicial en el desarrollo de la educación a distancia, a una segunda fase en la regulación por el cual se habilitan ofertas $100 \%$ virtuales, es dable suponer y proyectar, nuevas etapas de regulación futuras. Las primeras dos generaciones de regulación incluyen a los docentes o tutores, en tanto que los MOOCs son ofertas sin existencia de tutores, también se constata la génesis de ofertas de enseñanza sin tutores bajo los MOOCs. El caso de Ecuador pudiera ser visto ello como la génesis de una evolución normativa futura que pudiera ser la quinta generación de la regulación como sugerimos más adelante.

Un intento de clasificación de las fases de las normativas de regulación de la educación a distancia, nos pudiera llevar a proyectar el siguiente escenario pasado y futuro.

1) Creación de universidades públicas en América Latina en Colombia, Venezuela y Costa Rica. (Primera generación). Habilitación institucional y de modalidad.

2) Regulación de modelos semipresenciales (Segunda generación). Habilitación de tipo de oferta en la modalidad. Sedes y docentes o tutores.

3) Normativas de habilitación de la oferta $100 \%$ virtual (Tercera generación). Habilitación de tipo de oferta en la modalidad. Docentes y tutores. 
4) Habilitación de ofertas transfronterizas (Cuarta generación). Convenios internacionales, acuerdos de integración o normas nacionales. Apertura de mercado.

5) Habilitación de ofertas MOOCs (Quinta generación). Habilitación de tipo de oferta en la modalidad. Sin sedes ni docentes.

Ello corresponde no a etapas sino a categorías centrales de la habilitación y de la orientación de la política pública.

La diversidad de modelos y de tipologías en la educación a distancia, y el funcionamiento bajo multimodalidades, y más intensamente cuando se habilitan recorridos articulados donde los estudiantes pueden pasar de una modalidad a otra fácilmente, establece más complejidades de la regulación en base a estándares mínimos. Ello por cuando las multimodalidades imponen estándares diferentes de regulación de la oferta y de los criterios de habilitación

Las ofertas virtuales, así como las ofertas automatizadas (MOOCs) en todos los casos acompañan la posibilidad de las ofertas semipresenciales y aumentan la diferenciación institucional y pedagógica de la educación a distancia, y concomitantemente constituyen una de las bases que facilita el aumento del acceso y de la cobertura a distancia. La continua expansión de la educación a distancia en la región se asocia en uno de sus componentes a la diferenciación institucional y pedagógica. Ello ocurre, tal como también se ha dado con la diferenciación institucional de educación presencial en la región. Así, es dable suponer, a medida que aumenta la aprobación de nuevos marcos de políticas de regulación atentas a nuevas generaciones de regulación, a mayores aumentos de la cobertura de la educación a distancia.

Los análisis de las normas muestran habilitaciones diferenciales por áreas de conocimiento y de trabajo, que de hecho imponen reservas de mercado para las ofertas de educación presencial. Esta situación se constata fundamentalmente en las áreas de salud o ingeniería. En el caso de Ecuador, las limitaciones son por áreas de conocimiento utilizando el CINE. Hay también escenarios confusos de limitaciones, como en 
Brasil, donde la Orden de Abogados de Brasil, no habilitaba a presentarse a los exámenes de certificación y habilitación profesional a egresados de programas a distancia. Al tiempo el Ministerio no habilitaba programas a distancia en el área de derecho, aun cuando no estaba en la norma. Actualmente hay procesos en trámite de habilitación para ofertas a distancia. Ello implica que no todas las limitaciones son de normativas, sino también políticas o ideológicas.

El avance normativo que hemos analizado desde la habilitación de la oferta semipresencial hacia ofertas $100 \%$ virtuales, está cambiando el panorama regional de la educación superior que actualmente tendría la siguiente configuración.

\section{Normas de licenciamiento de la educación a distancia en América Latina}

\begin{tabular}{ll}
\hline Habilitación de Ofertas solo semi-virtuales. & Habilitación de Ofertas $\mathbf{1 0 0 \%}$ virtuales. \\
$\begin{array}{l}\text { Segunda Generación de regulaciones de } \\
\text { educación a distancia }\end{array}$ & $\begin{array}{l}\text { Tercera generación de regulaciones de } \\
\text { educación a distancia }\end{array}$ \\
\hline - Bolivia (privadas) & - Brasil (desde el 2017) \\
- Rep. Dominicana (privadas) & - Argentina (desde el 2017) \\
- Uruguay (privadas) & - México \\
- Perú & - Guatemala \\
- Panamá & - El Salvador (privadas desde el 2012) \\
& - Costa Rica (privadas desde el 2010) \\
& - Paraguay (desde 2016) \\
& - Ecuador (Desde el 2013) \\
& - Rep. Dominicana (pública) \\
\hline
\end{tabular}

Sin embargo, no todos los países siguen los mismos derroteros, sino que como hemos analizado comparativamente, hay caminos fuertemente diferenciados. Incluso, destaca el caso de Perú que marcharía a contra tendencia, el regular un modelo semipresencial de un máximo de $50 \%$, así como modelos de regulación con mayor libertad de oferta como Guatemala o que fueron anteriores como en México o Colombia. 
Los nuevos marcos normativos, destacan también particularmente, por cuanto dan mayor flexibilidad en la gestión y facilitan la estructuración de acuerdos de terciarización y de alianzas entre las instituciones, especialmente en la gestión de los centros de apoyo. Las rígidas políticas de regulación y de funcionamiento de los centros de apoyo se abandonan hacia modelos más flexibles. En esta orientación, los centros de apoyo se van diferenciando ajustados a los tipos de ofertas disciplinarias y profesionales. Los modelos de franquicias en la gestión de los centros de apoyo se verifican en las legislaciones de Brasil y Argentina. A medida que se habilita la oferta virtual, y por lo tanto se facilitan ofertas transfronterizas, se flexibiliza la localización de los centros de apoyo y de sus formas de fiscalización. En tal sentido, se destaca en algunos de los nuevos marcos, especialmente en Ecuador y Brasil, la incorporación de componentes internacionales en la regulación, y especialmente en lo referido la apertura de centros de apoyo fuera del país. Es esta a su vez la génesis de políticas normativas en materia de educación transfronteriza, y que hemos definido como de cuarta generación, y que marginalmente se comienzan a manifestar en la región.

\section{Conclusiones y recomendaciones}

En el inicio en América Latina se establecieron modelos educativos a distancia unimodales de tipo semipresenciales, estableciéndose entre otros componentes la obligatoriedad de momentos presenciales en el proceso de enseñanza, fundamentalmente en la evaluación (Mena, 2008). Sin embargo, con la digitalización, se comenzaron a crear modelos semivirtuales y virtuales que mostraron un corrimiento lento de la demanda hacia los nuevos modelos. En los últimos años se ha ido generalizando la habilitación de la oferta virtual. Ellas han incluido en general regulaciones con mínimos diferenciados de oferta para las distintas tipologías de educación a distancia. Así, los programas y ofertas de educación a distancia y sus modelos específicos, son una relativa derivación de esos 
marcos normativos de autorización de los programas, dependiendo sus especificidades del grado de flexibilidad y de la cantidad de variables o requisitos establecidos.

En el contexto de las innovaciones tecnológicas y educativas, y de la búsqueda de aumentar la demanda, se están conformando ofertas universitarias multimodales en educación a distancia, como resultado de la habilitación de ofertas 100\% virtuales o en línea, y la flexibilización de las tradicionales rigideces de la educación semipresencial que han derivado en rápidas virtualizaciones tipo "blended learning". El análisis regional a partir de algunos países que han aprobado nuevas normativas de regulación muestra como están aumentando la cantidad de países que habilitan las ofertas en línea, conformándose dinámicas basadas en la existencia de ofertas bajo multimodalidades (presencial, semipresencial y virtual). Ellas sin embargo mantienen múltiples diferenciaciones en términos de limitación y restricción de algunas ofertas académicas tanto sea por áreas de conocimiento o de niveles de enseñanza, o de flexibilidad de articulación, intensidad de los cursos, características de los centros de apoyo o formas de su terciarización y control del aseguramiento de la calidad.

Los nuevos escenarios de esta tercera generación de políticas de regulación de la educación a distancia parece estar llevando a la irrupción de nuevas formas de evaluación y acreditación, que superan las formas tradicionales de revisión documental, el cumplimiento numérico de indicadores de insumos y procesos, para incluir la evaluación de los resultados del aprendizaje de los estudiantes. Los antecedentes de México y Colombia, reafirman esta hipótesis

La habilitación de la oferta virtual y la existencia de multimodalidades, dadas por ofertas presenciales, a distancia y virtual, tienen múltiples definiciones y nombres, y puede ser considerada como un proceso en curso en la región, y estará en la base del aumento de la cobertura futura de la educación a distancia. Probablemente más países irán aprobando nuevos marcos de regulación de la educación a distancia de tercera generación, y abriendo caminos a las siguientes generaciones de políticas de regulación que se focalizan más en las TIC y la internacionalización. Ello 
sin embargo no implicará una regulación homogénea a escala regional sino que se mantendrá la alta diversidad. La virtualización de la educación a distancia como derivación de la tercera generación de políticas de regulación, visualiza nuevas fases futuras de regulación, algunos de cuyos componentes ya encuentran su génesis en algunas de las nuevas normativas desarrolladas desde el 2010.

\section{Bibliografía}

BATES, T. Cómo gestionar el cambio tecnológico. Estrategias para los responsables de centros universitarios (Ediuoc-Gedisa), Barcelona, 2000.

CLARK, B. El sistema de educación superior. Una visión comparativa de la organización académica. Nueva Imagen, Universidad Autónoma Metropolitana, México, 1991.

CLARK, B. Creando universidades innovadoras. 2000.

CONSEJO DE EDUCACIÓN SUPERIOR (CES). Reglamento de Régimen Académico. RPC-SE-13-No.051-2013, CES, Quito, 2013. Disponible en: <http://www.ces. gob.ec/gaceta-oficial/reglamentos >. Revisión, 14 julio, 2015.

CORREA, C. "Historia y tendencias de la educación a distancia en el Ecuador", en Morocho, Mary y Rama, Claudio (editores, "La universidad a distancia y virtual en Ecuador. Una nueva realidad universitaria” UTPL, CALED, Virtual Educa, 2013.

EL Horizon Report 2015 Higher Education Edition. Disponible en: <http://cdn. nmc.org/media/2015-nmc-horizon-report-HE-EN.pdf>.

GARCÍA ARETIO, L. ¿Por qué va ganando la educación a distancia? Madrid, UNED, 2009.

GARCÍA ARETIO, L. (coord.) De la educación a distancia a la educación virtual. Barcelona, Ariel, 2007. 
MENA, M.; RAMA, C.; FACUNDO, A. (compiladores). El Marco Regulatorio de la Educación Superior a Distancia en América Latina y el Caribe. UNAD, Virtual Educa, ICDE, 2008. Disponible en: <http://www.uned.es/catedraunescoead/cosypedal/ Marco\%20regulatorio\%20EaD\%20AL\%20-\%20Rama.pdf .

LEMAITRE, M. J. “Autoevaluación y Acreditación en el marco del aseguramiento de la calidad en el contexto Latinoamericano". Seminario Taller "Autoevaluación y Acreditación en el marco de la calidad", 7-9 feb., SINAES San José, Costa Rica, 2005. Disponible en: <http://acfo.edu.co/educacion/pdf/CALIDAD/calidad\%20 y\%20aeval\%20lemaitre.pdf $>$.

LUPION TORRES, P.; VIANNEY, J.; ROESLER, J. "Educación superior a distancia en Brasil”. En: LUPION TORRES, P.; RAMA, C. La Educación Superior a Distancia en América Latina y el Caribe Realidades y tendencias. UNISUL, Florianópolis, 2010. Disponible en: <http://virtualeduca.org/documentos/observatorio/oevalc_2010_(tendencias).pdf>.

MILARET, G., VIDAL, J. Historia mundial de la educación. Buenos Aires: Universidad de Palermo, 2010.

RAMA, C. La reforma de la virtualización. El nacimiento de la educación digital. Universidad de Guadalajara. Guadalajara, 2012. Disponible en: <http://virtualeduca.org/documentos/observatorio/libro_la-reforma-de-la-virtualizacion-de-la-universidad-claudio-rama-udg-2012.pdf >.

RAMA, C. La Tercera Reforma de la Educación Superior en América Latina. Fondo de Cultura Económica (FCE), Buenos Aires, 2006.

RODRÍGUEZ OSTRIA, G. De la revolución a la evaluación universitaria; cultura, discurso y políticas de educación superior en Bolivia. PIEB, La Paz, 2000.

RODRÍGUEZ RUIZ, J. R. "Aproximaciones a la educación a distancia en el Perú". En: DOMÍNGUEZ GRANDA, J. RAMA, C.; RODRÍGUEZ RUIZ, R. (editores). La educación a distancia en el Perú. Universidad Católica Los Ángeles de Chimbote, Virtual Educa, 2013. 
RUEDA (Red Universitaria de Educación a Distancia). CIN. Informe Técnico de Carreras de grado y Posgrado a Distancia de la República Argentina - 2015 Abril 2016. Disponible en: <http://rueda.edu.ar/wp-content/uploads/2017/07/ Informe_CarrerasEAD_Arg.pdf $>$.

SANTÁNGELO, H. "La normativa de Educación a Distancia para la enseñanza universitaria en Argentina: historia, aspectos relevantes y problemas". En: Mena, Marta, Rama, Claudio y Facundo, Ángel, en El Marco Regulatorio de la Educación Superior a Distancia en América Latina y el Caribe. UNAD; Virtual Educa. Bogotá, 2008.

Recibido: 10/06/17

Recebido: 10/06/17

Aprobado: 29/07/17

Aprovado: 29/07/17 